S.D. Ryder, D.J. Pisano, M.A. Walker, and K.C. Freeman, eds.

\title{
Galaxy Scaling Relations as a Result of Secular Evolution
}

\author{
Xiaolei Zhang \\ US Naval Research Laboratory, 4555 Overlook Ave SW, Washington, \\ DC 20375, USA
}

\begin{abstract}
The secular evolution process which slowly transforms the morphology of a given galaxy over its lifetime through mostly internal dynamical mechanisms could naturally account for most of the observed properties of physical galaxies (Zhang 2003a). As an emerging paradigm for galaxy evolution, its dynamical foundations had been established in the past few years (Zhang 1996, 1998, 1999). In this paper, we explore further implications of the secular morphological evolution process in reproducing the well-known scaling relations of galaxies.
\end{abstract}

\section{Disk Galaxy Scaling Relations}

A typical galaxy remains in a quasi-equilibrium configuration during the secular evolution process. From the Virial theorem relation $V^{2}=G M_{d y n} / R$, where $M_{d y n}$ is the dynamical mass of the galaxy. and the definition of average surface brightness $S B=L / R^{2}$, where $\mathrm{L}$ is the luminosity, it follows that $L \propto V^{4} \frac{1}{S B} \frac{1}{\left(M_{d y n} / L\right)^{2}}$, In order to have a tight Tully-Fisher relation $L \propto V^{4}$ (Tully $\&$ Fisher 1977), we must have $S B\left(M_{d y n} / L\right)^{2} \approx$ constant. The secular evolution process maintains the scaling relations by a decrease in galaxy's dynamical-massto-light ratio as the surface brightness of the galaxy increases during its Hubble type transformation (Zhang 2003b).

The fundamental plane relation for spirals can likewise be derived from the Virial theorem, resulting in $10 \log V=-(1+2 \beta) M_{t}-S B+$ constant, where $M_{t}$ is the absolute magnitude and $S B$ is the average surface brightness in magnitude/ $\operatorname{arcsec}^{2}$, and where $M_{d y n} / \mathrm{L} \propto L^{\beta}$. Pharasyn et al. (1997) found that fitting I band and $\mathrm{K}$ band data of a sample of spiral galaxies to this relation resulted in $\beta \approx-0.15$.

\section{Elliptical Galaxy Fundamental Plane Relations}

One of the many forms of the elliptical galaxy fundamental plane relation can be expressed as $L \sim V_{e}^{3.45} S B^{-0.86}$, or $M\left(R_{e}\right)=-8.62(\log V+0.1 S B)+$ constant (Djorgovski \& Davis 1987), where $M\left(R_{e}\right)$ refers to the absolute magnitude inside effective radius $R_{e}$. A slight rearrangement of the terms in this equation leads to $10 \log V=-1.25 M\left(R_{e}\right)-S B+$ constant. We see that apart from the constant term, the only difference between the spiral and elliptical fundamental plane relations is in the different signs of the mass-to-light ratio exponent $\beta$ : $\beta=-0.15$ for spirals and $\beta=0.13$ for ellipticals and bulges. As the secular evolution 
proceeds (which generally leads to an increase in L), the dynamical mass-to-light ratio of a spiral galaxy decreases because a larger fraction of the baryonic dark matter becomes luminous. On the other hand, elliptical galaxies are in more advanced stages of evolution and will experience a greater degree of dimming, which is reflected in the increase of dynamical mass-to-light ratio with $\mathrm{L}$.

\section{The Evolution Trend Revealed by the Three Types of Scaling Relations}

From the above three types of scaling relations, we can easily derive the following variations of the dependence of $\mathrm{M}_{d y n} / \mathrm{L}$ on mass and on light, and the interdependence of $\mathrm{M}_{d y n}$ and $\mathrm{L}$ :

(1). The dependence of mass on luminosity: $M_{d y n} \sim L^{0.5}$ from spiral Tully-Fisher relation, $M_{d y n} \sim L^{0.85}$ from spiral fundamental plane relation, $M_{d y n} \sim L^{1.12}$ from elliptical fundamental plane relation.

(2). The dependence of $\mathrm{M}_{d y n} / \mathrm{L}$ on luminosity: $M_{d y n} / L \sim L^{-0.5}$ from spiral Tully-Fisher relation, $M_{d y n} / L \sim L^{-0.15}$ from spiral fundamental plane relation, $M_{\text {dyn }} / L \sim L^{0.13}$ from elliptical fundamental plane relation.

(3). The dependence of $\mathrm{M} / \mathrm{L}$ on dynamical mass: $M_{d y n} / L \sim M_{d y n}^{-1}$ from spiral Tully-Fisher relation, $M_{d y n} / L \sim M_{d y n}^{-0.18}$ from spiral fundamental plane relation, $M_{d y n} / L \sim M_{d y n}^{0.1}$ from elliptical fundamental plane relation.

A gradual evolution trend of galaxy properties is thus implied in the three types of scaling relations. The fact that the $\mathrm{M}_{d y n} / \mathrm{L}$ ratio obtained from the spiral galaxy fundamental plane relation lies intermediate between that given by the spiral galaxy Tully-Fisher relation and the elliptical galaxy fundamental plane relation is apparently due to the fact that within the spiral fundamental plane relation there is the additional surface brightness parameter. The spiral fundamental plane effectively samples disk galaxy properties further inward, compared to the Tully-Fisher relation which samples characteristics averaged over the whole disk. More detailed discussion of the relation of the secular evolution process to the generation of galaxy structural properties and scaling relations can be found in Zhang (2003b).

\section{References}

Djorgovski, S. \& Davis, M. 1987, ApJ, 313, 59

Pharasyn, A., Simien, F., \& Heraudeau, Ph. 1997, in Dark and Visible Matter in Galaxies, eds M. Persic \& P Salucci (San Francisco: ASP), 180

Tully, R.B., \& Fisher, J.R. 1977, A\&A, 54, 661

Zhang, X. 1996, ApJ, 457, 125

Zhang, X. 1998, ApJ, 499, 93

Zhang, X. 1999, ApJ, 518, 613

Zhang, X. 2003a, astro-ph/0301655

Zhang, X. 2003b, ApJ submitted 